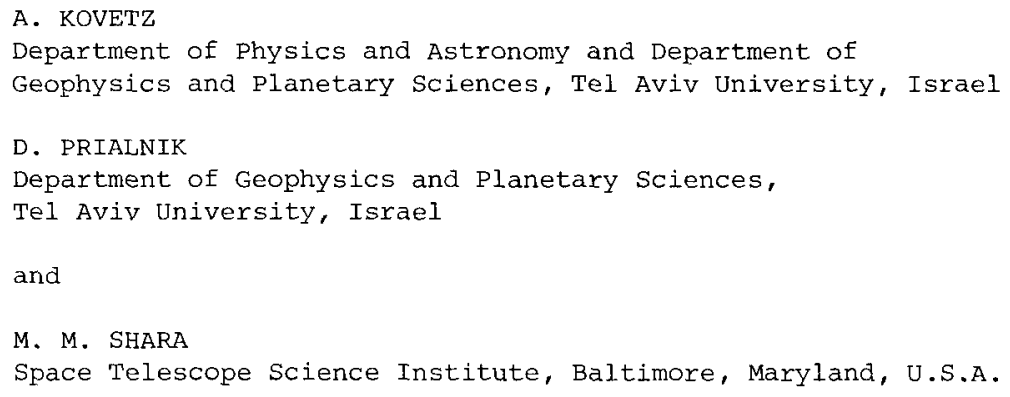

An evolutionary scenario for classical novae is proposed, which is intended to solve the discrepancies that exist between theory and observations:the space densitles of classical novae deduced from surveys in the solar neighbourhood are lower by about two orders of magnitude than those predicted theoretically, and the mass transfer rates in nova binaries, as estimated from observed luminosities in quiescence, are higher than those allowed by the thermonuclear runaway model for nova outbursts. These discrepancies disappear if mass transfer (at a high rate) takes place for only a few hundred years before and a few hundred years after an eruption, but declines afterwards and remains off for most of the time between outbursts. We show that such a behavior is to be expected if one takes into account the variation of binary separation, due to mass ejection on the one hand and angular momentum losses on the other hand.

Paper presented at the IAU Colloquium No. 93 on 'Cataclysmic Variables. Recent Multi-Frequency Observations and Theoretical Developments', held at Dr. Remeis-Sternwarte Bamberg, F.R.G., 16-19 June, 1986. 
One of the aspects of this scenario, on which we report in more detail, is the possibility of enhanced Roche-lobe overflow of the secondary, due to its expansion that results from irradiation by the high nova luminosity. We followed the evolution of a $0.5 \mathrm{M}$ main sequence star illuminated by a changing flux, typical of a classical nova. The numerical results indicate that, in spite of the slight binary separation that may occur after eruption, mass loss from the irradiated and thus bloated secondary should continue for a few hundred years. Other aspects of the variable $\dot{M}$ scenario are briefly summarized.

\section{INTRODUCTION: STATEMENT OF THE PROBLEM}

The goal of the present paper is to attempt to resolve two apparent discrepancies between observations of classical novae (CN) and the thermonuclear runaway (TNR) model for novae, which has otherwise achieved remarkable success in explaining a wide variety of eruption observations:

1. As Patterson (1984) has recently shown, the surveyed space density of $\mathrm{CV}$ binaries in general $\left(610^{-6} \mathrm{pc}-3\right)$ and $\mathrm{CN}$ in particular $\left(\sim 410^{-7} \mathrm{pc}^{-3}\right)$ appears to be much lower than the value deduced by applying nova theory to the observed eruption frequency of novae in the Galaxy and in M31 $\left(2-610^{-4} \mathrm{pc}^{-3}\right.$, Bath and Shaviv, 1978).

2. The mass transfex rate from the red dwarf to the white dwarf (WD) in nova binaries deduced by Patterson (1984) from accretion disk models (Tylenda, 1981) and observed CV luminosities is $\dot{M} \approx 10^{-7}-10^{-8} M_{\sigma} / y r$ (though, possibly as low as $10^{-9} M_{\circ}$ /yr in a few cases). But for hydrogen burning to occur in a sufficiently degenerate environment, to yield an explosive TNR followed by mass ejection, theoretical nova models and numerical 
simulations require an accretion rate $\dot{M} \leq 10^{-9} \mathrm{M}_{\odot} / \mathrm{yr}$ (Kutter and Sparks, 1980; Prialnik et al 1982, Fujimoto, 1982).

Nova models, however, have always assumed a constant $\dot{M}$. Invoking a variable mass transfer rate, Shara et al (1986) have suggested that theory and observations may be reconciled. Some of the problems involved in this new scenario are discussed below.

\section{THE CHANGE IN MASS TRANSFER RATE DURING A NOVA CYCLE}

During a nova eruption an amount of mass of about $10^{-5}-10^{-4}$ $M_{\odot}$ is ejected by the WD progenitor. Shara et al (1986) have shown that in spite of some angular momentum losses (MacDonald 1986), the binary system's separation, as well as the orbital period and the secondary's Roche lobe radius, increase (by a few $10^{-5}$, as the gravitational force between the stars decreases. strong observational support that mass loss tends to force the stars apart more than angular momentum losses draw them together is given by Schaefer and Patterson (1983), who found that the eclipsing nova BT Mon's orbital period increased by forty parts per million, as a result of the eruption. Even such a small increase in the separation of the $M$ dwarf and the WD can detach the former from its Roche-lobe and thereby reduce the mass transfer rate. This effect may, however, be delayed for some time, due to the radiation absorbed by the $M$ dwarf during the nova outburst, which should cause it to expand beyond its normal mainsequence radius. Thus old novae, for perhaps decades after eruption, can have high accretion rates. Only after it has cooled, may the secondary shrink within its Roche-lobe and stop 
transferring mass. The question we address in the next section is: how does a low mass main-sequence star respond to irradiation from a close nova outburst? In particular, on what timescale does it regain its former radius?

When mass transfer has almost turned off, the system's luminosity is due no more to accretion, but rather to the $M$ dwarf or the WD. Such a faint system should not be blue or exhibit emission lines and might easily escape CV surveys. Novae may spend most of their lifetimes in this hard-to-detect hibernation phase; CK Vulpeculae (Nova 1670) (Shara et al. 1985) is a likely candidate for a hibernating nova and WY Sge (Nova 1783) (Shara et al 1984) may be in a transition state between high and low $\dot{M}$. Thus the first conflict between theory and observations would be resolved.

During the long hibernation phase, when $\dot{M} \lesssim 10^{-12} \mathrm{M} / \mathrm{yr}$, gravitational radiation and/or magnetic braking by a stellar wind decrease the binaxy separation until Roche-lobe contact is restored. The material accreted by the WD after the nova outburst from the irradiated bloated secondary cools, becomes degenerate and diffuses slowly into the $\mathrm{C}-\mathrm{O}$ core. When mass transfer resumes, presumably at a fairly rapid rate $\left(M \sim 10^{-8} M_{0} / y r\right)$, the critical envelope mass required for a TNR will be achieved in $\leq$ $10^{3}$ years. Will the break in mass transfer permit a violent, mass ejecting eruption, despite the high $\dot{M}$ ? This question will be addressed in the last section of this paper. If the answer is yes, then indeed pre-novae should be as bright as post-novae, since both are in a state of relatively high mass transfer rate. 
It is still unclear, though, why the observed luminiosities of pre- and post-novae are so remarkably simil ar (Robinson, 1975), implying practically equal mass transfer rates, in spite of the differences in binary characteristics before and after an outburst.

\section{THE EFFECT OF IRRADIATION ON THE NOVA COMPANION}

We assume the secondary to be a 0.5 M main sequence star uniformly illuminated by a flux $\mathrm{S}=\sigma \mathrm{T}_{\mathrm{S}}^{\mathrm{H}}$ which varies with time as the nova luminosity (adopted from Prialnik 1986). Fig. 1 shows $L=4 \pi k^{2} \mathrm{~S}$, where $\mathrm{R}$ is the secondary's radius. To obtain the temperature distribution $T(\tau)$ as a function of the optical depth, we consider a flux $S=\sigma T_{s}^{4}$ that impinges normally on a semi-infinite medium. At all depths $\tau$, we distinguish between the beam $S e^{-T}$ which, though attenuated, retains its individuality, and the intensity of radiation $I(\tau, \mu)$ (in the direction $\theta=\cos ^{-1} \mu$ ), other than that contained in the beam. Then, for radiative equilibrium we obtain (in standard notation):

$$
\int I d \omega+S e^{-\tau}=4 \pi B
$$

Integration of the equation of transfer:

$$
\mu \mathrm{dI} / \mathrm{d} \tau=I(\tau, \mu)-B(\tau)
$$

and use of eq. (1) yields:

$$
\int \operatorname{I\mu d}\left(\boldsymbol{u}=\mathrm{Se}^{-\top}+\mathrm{T}\right.
$$

where $F$ is a constant. Since at any $\tau$, fIudw is the flux of radiation other than that in the beam, eq. (3) states that, apart from an amount $s e^{-\tau}$ required to cancel the beam flux, this flux, which represents the net radiation flux (in either direction), is constant with respect to $\tau$. 


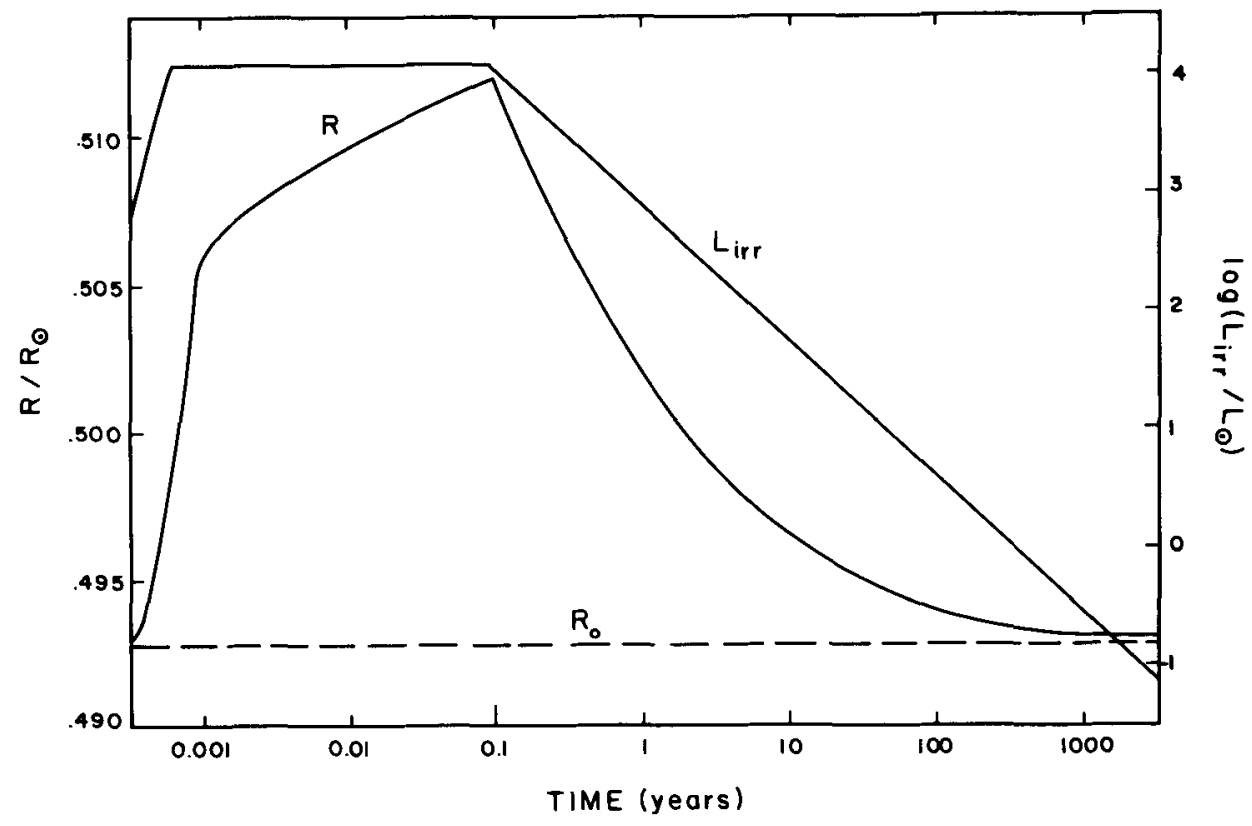

Fig.1 Variation of the irradiation luminosity $L_{\text {irr }}=4 \pi R^{2} S$ and its effect on the star's radius $R$ on a logarithmic time scale. Irr The star's main-seguence radius is $R_{0}$.

An approximate solution is obtained by setting:

$$
B(\tau)=a-b e^{-\tau}+c \tau
$$

where the constants $a, b$ and $c$ are derived by requiring radiative equilibrium and correct flux both at $\tau=0$ and at great depth $\tau+\infty$. This is an improvement over a similar approximation by Milne $(1930)$.

The result is:

$F=\left[\sigma T^{4}(\tau)-1.4427\left(1-\frac{1}{2} e^{-\tau}\right) \sigma T_{S}^{L}\right] /\left(\frac{3}{4} \tau+0.61067-0.18034 e^{-\tau}\right)$ 


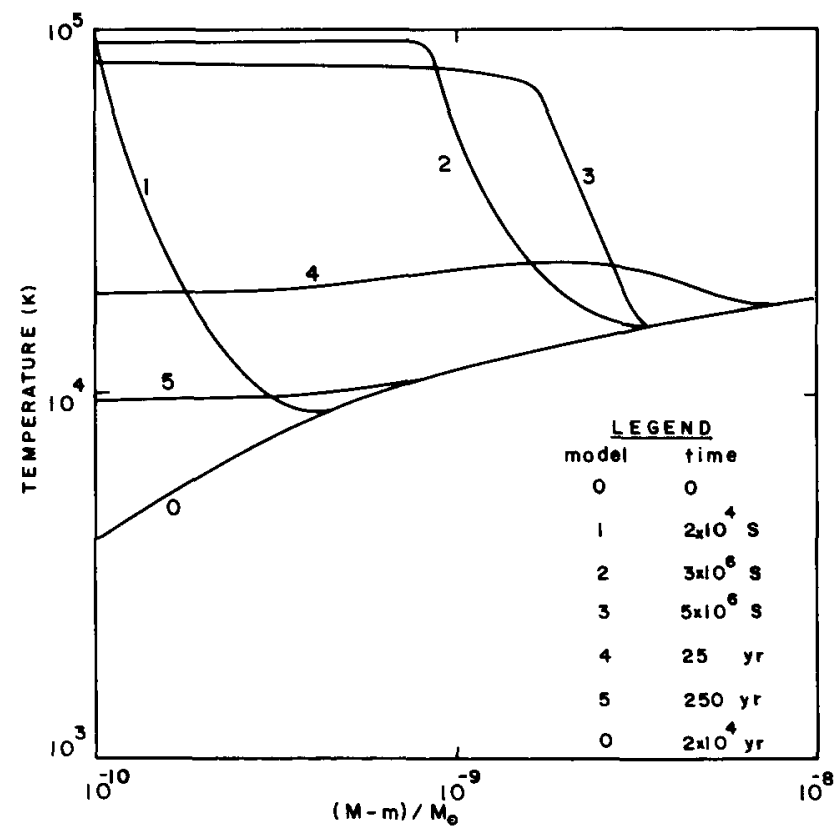

Fig.2 Temperature profiles over the outermost $10^{-8} \mathrm{M}_{\odot}$ of the irradiated star at different times during the $\Theta$ evolution, as indicated.

We note that at large $\tau, \sigma \tau^{4}(\tau)+\frac{3}{4} \mathrm{~F} \tau$, hence $F=a\left(\frac{1}{3} \mathrm{ar}^{2}\right) / \mathrm{d} \tau$ where $a=40 / \mathrm{c}$. The star's luminosity is:

$$
L=L_{i r r}+4 \pi R^{2} F
$$

The effect of irradiation on the secondary star is described in Fig. 1-3. Fig. 1 shows the variation with time of the star's radius. We note that the return to the main-sequence radius $R_{0}$ occurs within a few hundred years. In Fig. 2 temperature profiles are given for different times over the outer stellar region, where most of the impinging radiation flux is absorbed.

In order to estimate the change in mass transfer rate to be expected for the changing $R$, we assume $R_{0}=R_{L}$ (the Roche-lobe 


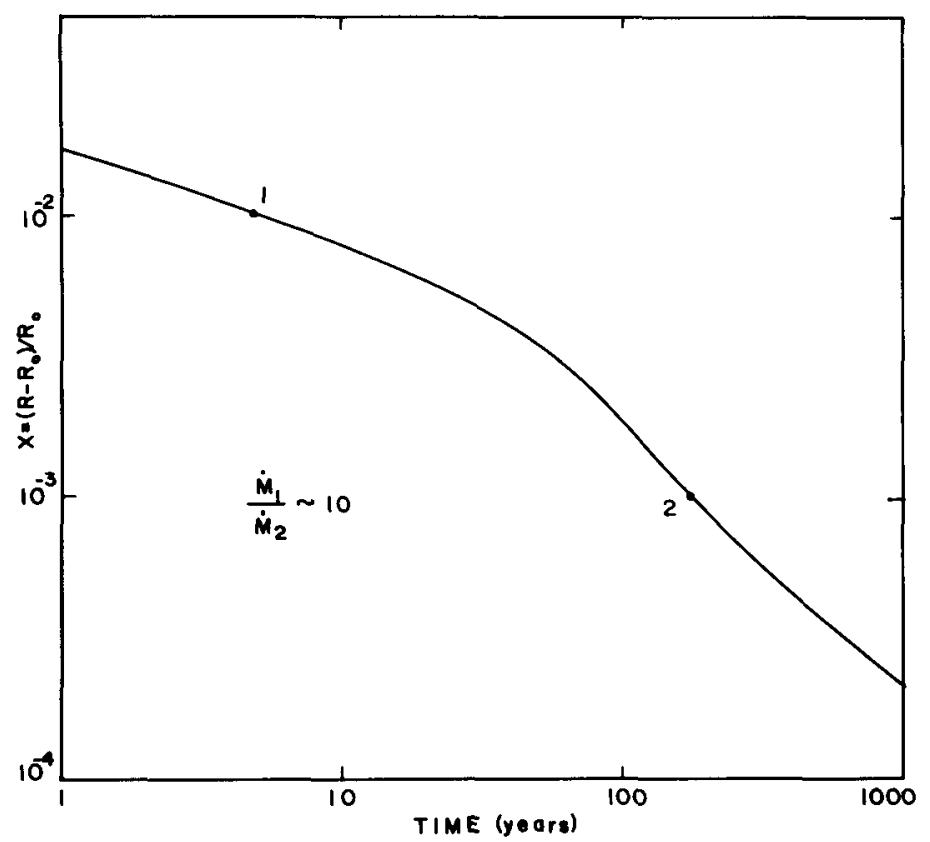

Fig. 3 Variation of the Roche-lobe overflow parameter $x$, The ratio between expected mass loss rates at points 1 . and 2 is indicated.

radius), define the Roche-lobe overflow parameter $x=\left(R_{-}-R_{0}\right) / R_{0}$ and adopt the approximation of Meyer and Meyer-Hofmeister (1983), which yields:

$$
\dot{\mathrm{M}}_{1} / \dot{\mathrm{M}}_{2}=\left[\mathrm{T}_{1} / \mathrm{T}_{2}\right]^{1.5} \mathrm{e}^{B\left(\mathrm{x}_{1}^{2} / \mathrm{T}_{1}-\mathrm{x}_{2}^{2} / \mathrm{T}_{2}\right)}
$$

where $B=\alpha G M U_{A} / 2 R_{L} R_{G}$, with $\alpha$ a coefficient of order 1 , depending on the Roche geometry, $M$ - the total mass, $\mu_{A}$ - the mean molecular weight and $\mathrm{R}_{\mathrm{G}}$ - the gas constant. The temperature $T$ is assumed constant between $R$ and $R_{0}$, an assumption which is amply fulfilled, according to Fig. 2. The 
variation of $x$ and the resulting change in $\dot{M}$ between two different points are shown in Fig. 3 .

We therefore conclude that, as a result of irradiation, the secondary will continue to transfer mass to the WD for $\geq 200$ years, during which $\dot{M}$ will decrease by about two orders of magnitude.

IV. NOVA MODELS OBTAINED WITH A VARIABLE $\dot{M}$

Numerical simulations were carried out by Prialnik and Shara (1986) to study the effect of two high $\dot{M}\left(=10^{-9} \mathrm{M}_{\odot} / y r\right)$ episodes, lasting centuries, separated by an inactive, or hibernation phase. The initial WD model was a $1.25 \mathrm{M}_{\odot} \mathrm{C}^{-O}$ star having a luminosity $L_{B O L}=5.610^{-3} L_{\odot}$. Models with hibernation times of $O(D), 10^{3}(\mathrm{C}), 10^{4}(\mathrm{~B})$, and $10^{5}(\mathrm{~A})$ years were calculated. As in previous simulations, continuous accretion (corresponding to zero hibernation time) at a rate $\dot{M}=10^{-P} \mathrm{M}_{0} / \mathrm{yr}$ produced a mild TNR, yielding a red giant rather than a nova. However, all three "hibernation" models experienced TNR sufficiently violent to eject mass at typical nova velocities, despite the fact that all the accreted mass was deposited at $\dot{M}=$ $10^{-8} \mathrm{M}_{\odot} / \mathrm{yr}$. The main results of the evolution through the hibernation phase are summarized in Table 1 and those concerning the outbursts are given in Table 2 .

In conclusion, the mass transfer rate relevant to the occurrence of a nova outburst is the effective (average) rate, which should not exceed a few $10^{-9} \mathrm{M}_{\odot} / \mathrm{yr}$, rather than the instantaneous rate, whether observed or undetected. 
TABLF 1

EVOLUTION THROUGH THE HIBERNATION PHASF

\begin{tabular}{|c|c|c|c|c|}
\hline Property & $\begin{array}{c}\text { (D) } \\
0^{*}\end{array}$ & $\begin{array}{c}\Delta t_{\text {hiberno }} \\
\text { (C) } \\
10^{3}\end{array}$ & ion $\begin{array}{c}(y x) \\
(B) \\
10^{4}\end{array}$ & $\begin{array}{l}\text { (A) } \\
10^{5}\end{array}$ \\
\hline $\mathrm{L}_{\mathrm{BOL}} / \mathrm{L}_{\mathrm{O}}$ & $5.42(-1)$ & $2.44(-2)$ & $7.75(-3)$ & $7.36(-3)$ \\
\hline $\mathrm{L}_{\mathrm{NUC}} / \mathrm{L}_{\mathrm{O}}$ & $2.22(-3)$ & $2.56(-5)$ & $\cdots$ & $\cdots$ \\
\hline$R\left(10^{-3} R_{0}\right)$ & 4.710 & 1.686 & 4.679 & 4.681 \\
\hline$T_{\text {eff }}(K)$ & $7.21\left(l_{t}\right)$ & $3.34 \quad(4)$ & $2,51(4)$ & $2.48(4)$ \\
\hline$Z_{\text {surface }}$ & $3.00(-2)$ & $2.89(-2)$ & $2.10(-2)$ & $2,15(-4)$ \\
\hline $\mathrm{Z}_{\mathrm{b}}^{* *}$ & 0.122 & 0.383 & 0.511 & 0.496 \\
\hline $\mathrm{T}_{\mathrm{b}}(\mathrm{K})$ & $1.48(7)$ & $1.05(7)$ & $8,4,0(6)$ & $8.21(6)$ \\
\hline$\rho_{b}\left(g \mathrm{~cm}^{-3}\right)$ & $2.20(3)$ & $3.16(3)$ & $3.62(3)$ & $3.79(3)$ \\
\hline$\varepsilon_{b}($ Fermi Parameter $)$ & 2.58 & 4.60 & 6.24 & 6.414 \\
\hline
\end{tabular}

*end of first accretion phase

** subscript $b$ denotes the base of the accreted matter

TABLE 2

OUTBURST AND RETATED CHARACTFRISTICS

\begin{tabular}{|c|c|c|c|c|}
\hline \multirow[b]{2}{*}{ Property } & \multicolumn{4}{|c|}{$M O D E L$} \\
\hline & A & B & c & D \\
\hline$M_{e f f}\left(M_{0} y x^{-1}\right)$ & $6.6(-11)$ & $6.4(-10)$ & $3.9(-9)$ & $1.0 \quad(-8)$ \\
\hline$\Delta m_{a c c} / M_{0}$ & $6.59(-6)$ & $6.88(-6)$ & $6,32(-6)$ & $4.99(-6)$ \\
\hline $\mathrm{m}_{\mathrm{env}} / \mathrm{M}_{\mathrm{O}}$ & $7.43(-6)$ & $7.30(-6)$ & $6.65(-6)$ & $5.26(-6)$ \\
\hline$\Delta m_{\text {ejecta }} / M_{0}$ & $3.79(-6)$ & $4.08(-6)$ & $4.63(-6)$ & $\cdots$ \\
\hline & $\left(51 \% \mathrm{~m}_{\mathrm{env}}\right)$ & $\left(56 \% \mathrm{~m}_{\mathrm{env}}\right)$ & $(70 \% \mathrm{menv})$ & \\
\hline $\mathrm{Z}_{\text {ejecta }}$ & 0.155 & 0.099 & 0,095 & $\cdots$ \\
\hline $\mathrm{T}_{\max }(\mathrm{K})$ & $2.287(8)$ & $2.245(8)$ & $2.123(8)$ & $1.876(8)$ \\
\hline $\mathrm{v}_{\max }(\mathrm{km} \mathrm{sec}-\mathrm{l})$ & 2940 & 2670 & 2670 & $\cdots$ \\
\hline
\end{tabular}




\section{References}

Bath, G.T. and Shaviv, G. 1978, M.N.R.A.S., 183, 515 .

Fujimoto, M.Y. 1982, Ap.J., 257, 752.

Kutter, G.S. and Sparks, W.M. 1980, Ap. J., 239. 988.

Mac Donald, J. 1986, Ap. J. in press.

Meyer, F. and Meyer-Hofmeister, E. 1983, Astr. Ap. 121, 29.

Milne, E.A., 1930, Handbuch der Astrophysik, Vol. 3, Part 1.

Patterson, J. 1984, Ap. J. Suppl. Ser. 54, 443.

Prialnik, D. 1986, Ap. J. in press.

Prialnik, D., Livio, M., Shaviv, G. and Kovetz, A, 1982, Ap. J. 257,312 .

Prialnik, D. and Shara, M.M., 1986, Ap. J. in press.

Robinson, E.L. 1975, A.J., 80,515.

Schaefer, B.E. and Patterson, J. 1983, Ap. J., 268, 710.

Shara, M.M., Livio, M., Moffat, A.F.J. and Orio, M. 1986, Ap. J. in press.

Shara, M.M., Moffat, A.F.J., McGraw, J.T., Dearborn, D.S., Bond, H.E., Kemper, E., and Lamontagne, R. 1984, Ap. J., 282, 763.

Shara, M.M., Moffat, A.F.J. and Webbink, R.N. 1985, Ap. J. 294, 286 .

Tylenda, R., 1981, Acta. Astr. 31, 127. 